\title{
Primary closure of open myelomeningocele
}

\author{
SARah J. Gaskill, M.D., M.D. \\ Pediatric Neurosurgery of South Texas, P.A., San Antonio, Texas
}

\begin{abstract}
Object. The purpose of this paper is to describe the technique of closing an open myelomeningocele in the newborn infant.

Methods. The anatomical rationale behind the closure techniques will be specifically reviewed. Avoidance of complications will be discussed. The management of unusual developmental anatomical abnormalities, such as the management of unusually large skin defects and kyphotic deformities will be discussed. Complications and the postoperative care will be reviewed and specifics regarding postoperative care will be summarized.
\end{abstract}

Conclusions. A multidisciplinary team approach is critical to the long-term management of these patients.

\section{Key Words • myelomeningocele • spina bifida • neural tube defect • spinal dysraphism • hydrocephalus • neural placode}

Myelomeningocele remains the most complex congenital malformation of the central nervous system that is compatible with life. This lesion results when the neural tube fails to fold normally during postovulatory Days 21 to $27 .{ }^{6}$ The exact cause of disorders remains under some historical debate and is not within the scope of this paper. Myelomeningocele within the context of this discussion refers only to lesions that involve an open caudal neural tube defect on the surface of the skin (Fig. 1). Within the category of spina bifida it affects approximately one in every 1000 live births, although there is clear geographical variability in the incidence of this congenital malformation. The debate regarding whether to close an open defect has long since been resolved. The purpose of this article is to detail the initial surgical closure of an open myelomeningocele defect, with attention to some of the more uncommon and unusual features that can be found at surgery. Each repair is unique and the anatomical variation is as great as the number of cases. Using embryological and developmental knowledge, an effective repair is possible in all cases. This paper is not intended to cover all of the variations of neural tube defects that occur such as diastematomyelia, lipomyelomeningocele, and myelocystocele.

If the diagnosis of myelomeningocele is made prenatally, counseling and a planned cesarean delivery are preferred. Optimally, the delivery will be scheduled in a center where there is a neurosurgeon who specializes in the care of children. Once a child is born with myelomeningocele a thorough physical examination must be conducted to look for associated conditions that in particular might affect the surgical procedure, such as pulmonary or cardiac abnormalities. Radiographic evaluation with cranial

Abbreviations used in this paper: $\mathrm{CSF}=$ cerebrospinal fluid; $\mathrm{VB}=$ vertebral body. and renal sonograms is important, but need not delay surgical intervention. Spinal films can be performed, but are not essential. The timing of surgery is important because there is an association with delay of surgery and an increase in the infection rate. In our institution we aim to close the lesion within 6 to 12 hours after birth unless there are medical conditions that would prevent administration of a general anesthetic and/or the surgical procedure.

After birth and prior to surgical intervention, the open myelomeningocele defect should be gently covered with sterile saline-soaked gauze. A kerlix wrapped gently around the torso and then soaked with sterile saline over the site is effective. The infant should be positioned prone or laterally to avoid pressure on the placode prior to surgery. Intubation after the induction of a general anesthetic should be performed with the infant in the lateral decubitus position or with the infant supported supine so there is no pressure on the defect. This is easily managed by cradling the child in the forearms laterally on the torso. A latex-free Foley catheter should be placed prior to surgery to simplify nursing care postoperatively. This allows the patient to be in the prone position postoperatively without the risk of bladder distention going unrecognized in the perioperative period.

In the operating room, the infant should be positioned on lateral rolls to prevent pressure on the abdomen. In cases in which there are significant orthopedic deformities of the legs, positioning can be a challenge, but can be accomplished with use of foam and pillows, even in cases with severe orthopedic deformities. The entire back, including the open defect, is then prepared with Hibiclens solution. Draping should allow maximal access to the back for the skin closure. Under magnification of a surgical microscope the surgery is begun. The defect is approached by separating the placode, which is a reddish tis- 


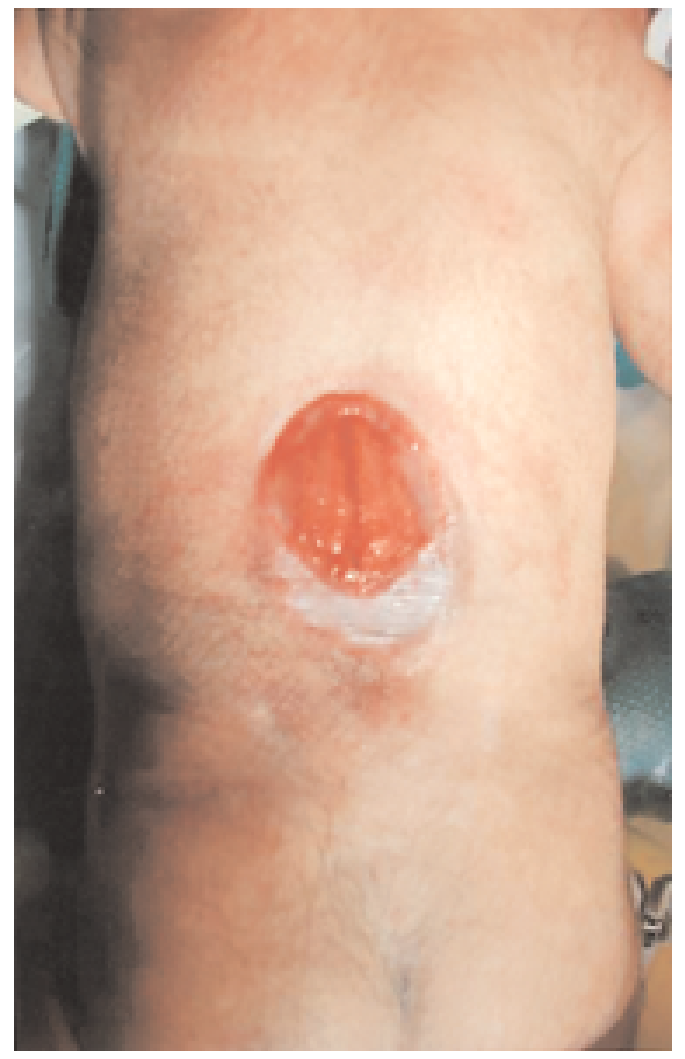

Fig. 1. Photograph showing an open myelomeningocele defect.

sue oriented in the caudal cephalad plane with a visible neural groove, from the intermediate zone. Swabs for aerobic and anaerobic cultures should be taken as soon as CSF is encountered. Antibiotic drugs are generally continued until the cultures from the placode are negative for 72 hours. Nerves exiting from the placode and terminating in the intermediate zone or laterally can be sacrificed because they are not functioning. All other nerves should be carefully preserved. The goals of separation of the placode are to reduce the possibility of future tethering and to enable reconstruction of the normal neural tube. Once the placode has been separated from the intermediate zone it should be carefully inspected under the microscope so as to remove any potential tissue that could form a dermoid cyst at a later date. All segments of intermediate tissue and skin must be removed from the placode itself. The placode can then be "reconstructed" into a tube by using a 5-0 silk suture. The suture is more easily drawn through the tissue with minimal damage if it is oiled with sterile mineral oil. It is important to be sure there is no tethering at the most cephalad aspect of the placode where the normal spinal cord ends. There is debate about resecting compared with reconstructing the placode. Certainly, with the goal of restoring the most normal anatomy possible, reconstruction is preferred. Additionally, Reigel, et al., ${ }^{9}$ demonstrated that intraoperative evoked potentials monitoring of the placode confirms intact afferent connections between the placode and the cortex. Therefore, if the placode appears to be in good condition, reconstruction is a preferable alternative.

Once the placode has been reconstructed, attention is turned to separating the dura from the epidural space. The dura is usually copious and if care is taken to make the separation at the most lateral margins of the defect, there is no problem with obtaining a good watertight seal when completing the dural closure. Once the dura has been separated, it should be reconstructed in a watertight fashion with 5-0 silk suture. Once again, using mineral oil on the suture aids in the closure. The closure should be tested with the infant's head elevated by placing the operating table in reversible trendelenburg and having the anesthesiologist induce a Valsalva maneuver. A watertight dural closure will reduce the risk of CSF leakage postoperatively and consequently reduce the incidence of tethered cord. Other than reducing the risk of CSF leakage, there is no known surgical technique at this time to help reduce the risk of future tethered cords. An experimental study in rabbits in which Poloxamer 407 (a nonionic block copolymer with both hydrophilic and hydrophobic chains and low dermal and mucosal toxicity) was used demonstrated a $50 \%$ reduction in leptomeningeal adhesions in surgical meningeal injury. ${ }^{8}$ Unfortunately, no further investigation has been reported. In short, no technological advances to aid in the long-term reduction of tethered cords have been reported in the literature.

In some cases, the open and malformed laminae prevent a good muscle and skin closure. In such cases, osteotomies at the base of the laminae can be easily performed using an Alice clamp attached at the base and forcing this medially, in effect making an osteotomy at the base of the pedicle, which is malformed in most cases. If malformed laminae cannot be repositioned into a useful anatomic position, then they should be rongeured to enable a good muscle closure.

In cases in which there is a significant kyphotic deformity, it is critical to perform a surgical correction at this time. In an extensive study by Crawford, et al., ${ }^{1}$ a retrospective review of 12 of 124 patients who underwent kyphectomy at the time of initial closure was performed. With one patient excluded because surgery was performed by a different surgeon, a total of 11 patients were followed for a mean of 7 years and 4 months. They found that the average preoperative kyphotic deformity measured $67^{\circ}$ with an average initial correction of $77^{\circ}$. Primary wound closure was possible in all cases, and there were no serious complications. Although there was some recurrence of the kyphosis with time, the deformities were mild and involved a more rounded and less severe deformity. Only one patient required repeated surgery for the deformity. At the time of the initial closure, the kyphotic vertebrae can be easily resected with rongeurs. The abnormal laminae and malformed processes associated with the resected VB should also be removed. Care taken to perform the resection directly against the VB and using meticulous hemostasis will help prevent significant blood loss. Using a gentle but steady downward pressure on the adjacent vertebrae to allow approximation, a 0-nuralon with a cutting needle is used to sew the adjacent segments together. This allows for good anatomical alignment of the remaining VBs. A tortoise shell orthosis for the spine with an opening over the incision will need to be made postoperatively and should be used for the 1st year of life with modifications made to accommodate growth.

The next layer to be addressed is the muscle closure. It 
is essential to achieve a good muscle closure in all cases. If the lesion is small, adequate muscle can often be obtained by making lateral releases in the paraspinal muscles on either side and approximating the muscle in the midline. For larger defects, or in those in which there is not enough lateral muscle, the muscle must be moved from above the level of the lesion. Extending the midline incision cephalad and retracting the skin will allow for adequate access to the muscle. The length of the defect is measured, and this guides the incisions made in the muscle. Starting approximately $2 \mathrm{~cm}$ off the midline, the paraspinal and latissimus dorsi muscles are divided. This is done on both sides, taking care to leave an adequate layer of muscle in situ to preserve the muscle function of the torso. The lateral incisions are connected across the midline and the cautery is used to take down the muscle from the spinous processes without making a hole in the layer of muscle in the midline. In this fashion, a sheet of muscle can then be flapped down over the entire defect and sewn in place with 2-0 vicryl. There are many reports of complex plastic surgical closures in the literature; $4,5,7,10$ however, at our institution we have found that closures of even the largest defects (Fig. 2) can be effectively performed by the neurosurgeon when using the above techniques.

Once the muscle has been closed, the skin is now examined to allow for a primary closure. The skin can be dis-

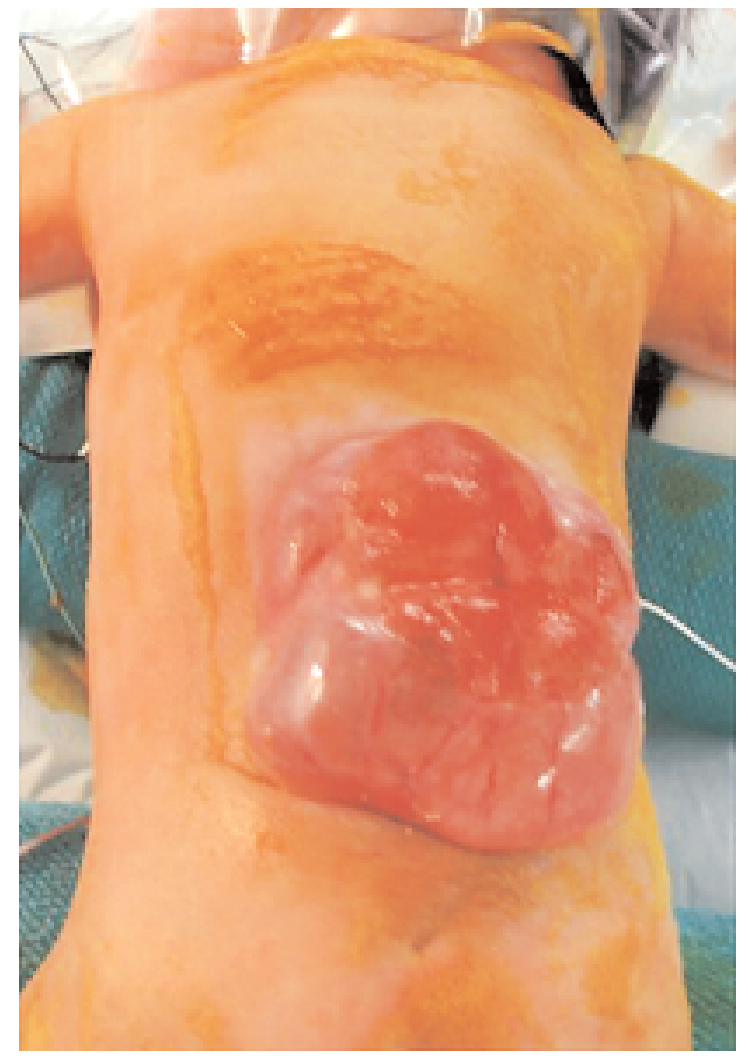

Fig. 2. Photograph showing a large open myelomeningocele defect, which was effectively closed with paraspinal and latissimus dorsi rotational flaps. sected bluntly from the underlying tissue laterally, caudally, and superiorly as needed. All intermediate and abnormal tissue should be excised from the skin margins. Skin hooks and temporary stitches can be used to help determine the optimal approximation of the skin edges. In most cases, the skin can be closed in a simple midline closure. In some cases, a T-type incision is required. A good closure is provided by 3-0 vicryl in the subcutaneous tissues with a running 4-0 prolene for the skin. An occlusive dressing with a protective layer of plastic can be fashioned from a pediatric urine collection bag (Fig. 3). ${ }^{2}$ The dressing is removed if it becomes soiled and/or on the 2 nd or 3rd postoperative day. At that time, wound care is begun with hydrogen peroxide and Neosporin three times daily.

Postoperatively the infant should be positioned prone while in the crib until the sutures are removed at 7 to 10 days. The infant can be held for feeding, nursing, therapy, and parental bonding. Care should be taken to avoid extended periods of pressure on the lateral trunk, in particular if the defect was large and the skin is taut. We have found the use of a Foley catheter in the immediate postoperative period to be a useful adjunct because urinary retention can develop in these infants and the prone position makes it difficult for the nursing staff to detect this. The catheter is usually left in place for several days and then urological care is deferred to the pediatric urologist. It also aids in the prevention of soiling the incision with urine.

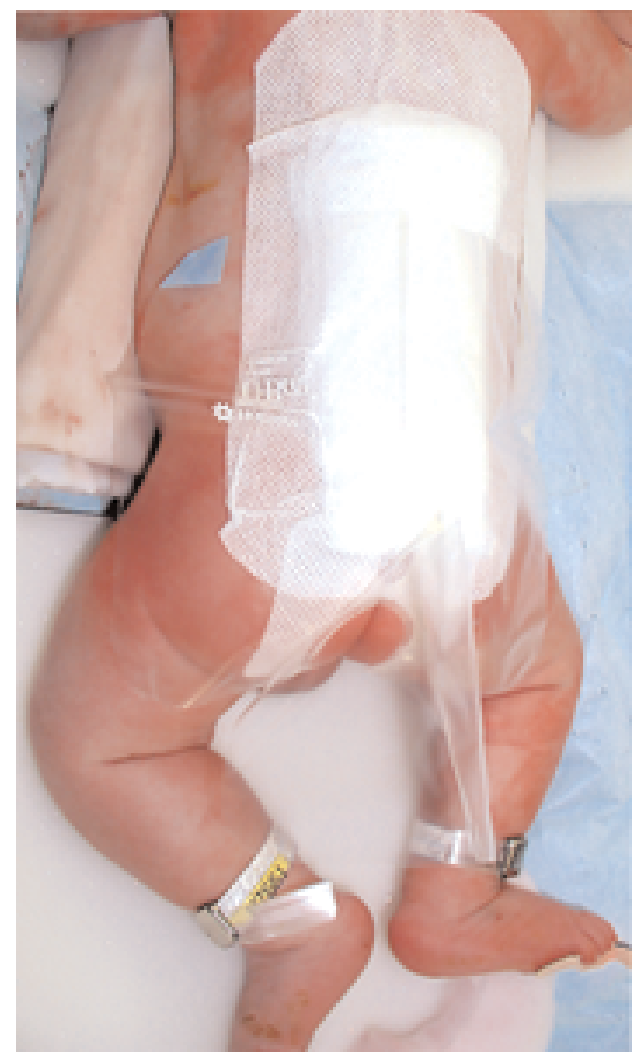

Fig. 3. Postoperative photograph showing the dressing applied including the urine bag cut to allow protection of the wound from fecal soiling. 


\section{COMPLICATIONS AND POSTOPERATIVE CARE}

The most common complications of closure of myelomeningocele are blood loss, CSF leak, infection, wound healing problems, and tethered cord. Blood loss is usually not sufficient to require a transfusion if careful hemostasis is maintained throughout the procedure. Cerebrospinal fluid leakage after achieving a good three-layer closure is uncommon. The infant should be observed closely for the development of hydrocephalus after closure of the open defect and shunt placement should be initiated when the diagnosis is clear. Infection is uncommon. Typically, antibiotic drugs are initiated prior to the repair and are maintained until cultures taken in the operating room are negative at 72 hours. Wound healing difficulties in particularly large wounds can occur, but are readily managed with good wound care and keeping the infant prone. For small wound breakdowns or openings in the skin incision, Preparation $\mathrm{H}$ should be applied three times daily. This has been shown to greatly enhance wound healing in our institution and in the literature. ${ }^{3}$ Unfortunately, no techniques have been demonstrated to reduce the incidence of postoperative spinal cord tethering and approximately 15 to $20 \%$ of children will develop a tethered cord at some point in their lifetime, which will require a repeated operation.

\section{CONCLUSIONS}

The multidisciplinary team is critical to the ultimate success and long-term management of these patients. The initial closure is just the beginning of the medical care that these children will require. An integrated team of neurosurgeons, orthopedists, urologists, physiatrists, therapists, and nurses is important to long-term care. Children born with myelomeningocele have the opportunity to live normal, healthy, and productive lives in the majority of cases.
Consistent and frequent long-term medical follow up is essential to achieving this goal.

\section{References}

1. Crawford AH, Strub WM, Lewis R, et al: Neonatal kyphectomy in the patient with myelomeningocele. Spine 28:260-266, 2003

2. Esckilsen A, Marlin AE, Gaskill SJ: Technical note: meningomyelocele dressings. Pediatr Neurosurg 29:280, 1998

3. Goldwyn RM: Preparation H. Plast Reconstr Surg 101: 509-510, 1998

4. Kitazawa T, Iswasawa M, Shinohara H: Extended latissimus dorsi musculocutaneous flap for closure of a meningomyelocele in a 5-hour-old neonate. Ann Plast Surg 47: 215-216, 2001

5. Lapid O, Rosenberg L, Cohen A: Meningomyelocele reconstruction with bilobed flaps. Br J Plast Surg 54:570-572, 2001

6. Mclone, DG, Dias MS: Normal and abnormal early development of the nervous system, in Cheek WR (ed): Pediatric Neurosurgery: Surgery of the Developing Nervous System, ed 3. Philadelphia: WB Saunders, 1994, pp 3-39

7. Munro IR, Neu BR, Humphreys RP, et al: Limberg-latissimus dorsi myocutaneous flap for closure of myelomeningocele. Childs Brain 10:381-386, 1983

8. Reigel DH, Bazmi B, Shih SR, et al: A pilot investigation of the poloxamer 407 for the prevention of leptomeningeal adhesions in the rabbit. Pediatr Neurosurg 19:250-255, 1993

9. Reigel DH, Dallmann DE, Scarff TB, et al: Intra-operative evoked potential studies of newborn infants with myelomeningocele. Dev Med Child Neurol Suppl 37:42-49, 1976

10. Sarifakioglu N, Bingül F, Terzioglu A, et al: Bilateral split latissimus dorsi V-Y flaps for closure of large thoracolumbar meningomyelocele defects. Br J Plast Surg 56:303-306, 2003

Manuscript received November 15, 2003.

Accepted in final form January 12, 2004.

Address reprint requests to: Sarah J. Gaskill, M.D., 4499 Medical Drive, Suite 397, San Antonio, Texas 78229. 